\title{
Radiographic Progression of Silicosis among Japanese Tunnel Workers in Kochi
}

\author{
Narongpon Dumavibhat ${ }^{1,2}$, Tomomi Matsui ${ }^{1}$, Eri Hoshino ${ }^{3}$, Sasivimol Rattanasiri ${ }^{4}$, \\ Dittapol Muntham ${ }^{4}$, Ryoji Hirota ${ }^{1}$, Masamitsu EitoKu ${ }^{1}$, Momo ImanaKA ${ }^{1}$, \\ Basilua Andre Muzembo ${ }^{1}$, Nlandu Roger NGatu ${ }^{1}$, Shinichi Kondo ${ }^{5}$, Norihiko Hamada ${ }^{6}$ \\ and Narufumi SUgANUMA ${ }^{1}$
}

${ }^{1}$ Department of Environmental Medicine, Kochi Medical School, Kochi University, Japan, ${ }^{2}$ Department of Preventive and Social Medicine, Faculty of Medicine Siriraj Hospital, Mahidol University, Thailand, ${ }^{3}$ Clinical Trial Center, Kochi Medical School, Kochi University, Japan, ${ }^{4}$ Section for Clinical Epidemiology and Biostatistics, Faculty of Medicine Ramathibodi Hospital, Mahidol University, Thailand, ${ }^{5}$ Department of Occupational Medicine, Kinro Clinic, Japan and ${ }^{6}$ Department of Radiology, Kochi Medical School, Kochi University, Japan

\begin{abstract}
Radiographic Progression of Silicosis among Japanese Tunnel Workers in Kochi: Narongpon DumaviBHAT, et al. Department of Environmental Medicine, Kochi Medical School, Kochi University, Japan-Objective: The aim of our study was to investigate the natural course of silicosis in terms of radiographic progression among Japanese tunnel workers. Methods: Tunnel workers with silicosis were included in our study between January 2008 and June 2011. We retrospectively assessed workers' radiographs from their first through last visits to see whether there was progression. All films were interpreted by two physicians, who had been specially trained in using the ILO (2000) International Classification of Radiographs of Pneumoconioses (ILO/ICRP). We classified the radiographic findings according to the ILO/ICRP. Survival analysis was performed and then presented as time to progression. Subgroup analysis among the progressed group was performed to demonstrate duration of progression. Results: A total of 65 patients, who were no longer exposed to silica for the duration of the study, were included. The mean age at the first visit was $58.60 \pm 7.10$ years. The incidence rate of progression was 42 per 1,000 person-years with a median time to progression of 17 years. Progression was demonstrated among 33 cases $(51 \%)$. The mean durations of progression from category 1 to category 4 and category 2 to category 4 were 14.55 and 10.65 years, respectively. Most patients $(86 \%)$ had radiographic change from category 1 or 2 directly to category 4 . Conclusion:
\end{abstract}

Received Nov 8, 2012; Accepted Feb 15, 2013

Published online in J-STAGE Mar 13, 2013

Correspondence to: N. Dumavibhat, Department of Environmental Medicine, Kochi Medical School 185-1, Kohasu, Oko-cho, Nankokushi, Kochi 783-8505, Japan (e-mail: dumavibhat@yahoo.com)
Silicosis progressed at a relatively high rate among tunnel workers without further silica exposure. The high probability of progression directly from category 1 to category 4 may lead to further investigation for the improvement of disease prevention.

(J Occup Health 2013; 55: 142-148)

Key words: Chest radiograph, ILO Classification, Pneumoconiosis, Progression, Silicosis, Tunnel worker

Silicosis, one of the oldest occupational illnesses, is a fibrogenic lung disease caused by inhalation of free crystalline silica ${ }^{1)}$. This incurable disease can progress even after silica exposure is discontinued. Consequently, it may lead to long-term disability or death during or after exposure to the dust. In addition, the International Agency for Research on Cancer (IARC) has classified crystalline silica as a group-1 carcinogen to humans ${ }^{2)}$. As a result, silicosis has taken a more significant place among the world's occupational health problems. Although considerable efforts have been employed to prevent silicosis, tens of millions of workers have been affected worldwide ${ }^{1)}$. Recently, the incidence of silicosis has decreased in countries where implementation of preventative measures has been successful. Nevertheless, there are still new cases every year because of long-term exposure to silica in the past. Therefore, more than 1,000 new patients with pneumoconioses, mainly silicosis and coal worker's pneumoconiosis, are compensated annually in industrialized countries in Asia, i.e., Japan and Korea ${ }^{3,4)}$.

To date, there are few longitudinal studies that 
have reported radiographic progression of the disease among workers in different occupational groups, such as sandblasters ${ }^{5)}$, slate pencil workers ${ }^{6}$, iron ore miners $^{7)}$, granite quarry workers ${ }^{8,9)}$, coal workers ${ }^{10)}$, whetstone cutters ${ }^{11)}$, and silica flour packers ${ }^{12}$. Tunnel workers are also one of the populations at risk for developing silicosis, but there has been no study about radiographic progression of the disease. Frequent relocation of workers may be the main reason for the difficulty in conducting longitudinal studies among tunnel workers. The aim of this study was to investigate the natural course of silicosis after termination of silica exposure in terms of radiographic progression among Japanese tunnel workers. The results of this study indicate the long-term consequences for tunnel workers with silicosis. Furthermore, the current situation in industrialized countries may reflect the future picture of silicosis among the tunnel workers in developing countries; therefore; this study will lead to more effective prevention.

\section{Materials and Methods}

Study population

We conducted a retrospective cohort study of tunnel workers with silicosis who lived in Kochi Prefecture and visited the Occupational Medicine Department at Kinro Clinic from January 2008 through June 2011. For all of these patients, diagnosis of silicosis had been confirmed by the Pneumoconiosis Panel of the Labor Office, according to the Pneumoconiosis $\mathrm{Law}^{13)}$. At the time patients began receiving treatment at the clinic, they discontinued their employment and then visited the clinic annually. Criteria for diagnosis of silicosis in the present study were a history of silica exposure, and chest radiography compatible with profusion of at least $1 / 0$ according to the ILO International Classification of Radiographs of Pneumoconioses ${ }^{14)}$. In addition, spirometry was used to evaluate severity for compensation purposes. Inclusion criteria were male tunnel workers who suffered from silicosis and were followed up at the clinic between January 2008 and June 2011. Furthermore, every patient was required to have at least a one-year follow-up and at least two chest radiographs to see whether there was any progression. We retrospectively reviewed all chest radiographs, computed tomography (CT) and medical records from their first to last visits. Demographic data, work history, smoking history and spirometry data were obtained from medical records. An IRB review was waived because of the retrospective and noninvasive nature of this study.

\section{Chest radiographic assessment}

Chest radiographs were interpreted by two readers, including one chest physician who was a National Institute for Occupational Safety and Health-approved $\mathrm{B}$ reader ${ }^{15}$ and one occupational medicine physician who was a certified Asian Intensive Reader of Pneumoconioses (AIR Pneumo) ${ }^{16,17)}$. Furthermore, computed tomography of the chest was used to confirm abnormal findings of chest radiographs in every case, referring to the International Classification of HRCT for Occupational and Environmental Respiratory Diseases (ICOERD) ${ }^{18,19)}$. The final decision was made by consensus if there was disagreement between the readers. The readers interpreted all chest radiographs using side-by-side assessment ${ }^{20)}$, in which they viewed all films together in known temporal order, and compared them with the ILO standard films. The ILO (2000) International Classification of Radiographs of Pneumoconioses was used to classify small and large opacities. The classification scheme for the profusion of small opacities utilizes a 4-point major category scale $(0,1,2$ and 3$)$, with each major category divided into three, giving 12 minor categories of increasing profusion $(0 /-, 0 / 0,0 / 1,1 / 0,1 / 1$, $1 / 2,2 / 1,2 / 2,2 / 3,3 / 2,3 / 3$ and 3/+). Extent of large opacities was categorized accordingly into $\mathrm{A}, \mathrm{B}$ and $\mathrm{C}$. Category A was defined as an opacity having a minimum longest dimension exceeding $1 \mathrm{~cm}$ but no more than $5 \mathrm{~cm}$ in length or several large opacities with the sum of their longest dimensions not exceeding 5 $\mathrm{cm}$. Category B was defined as a single opacity or as multiple opacities with the sum of their longest dimensions greater than $5 \mathrm{~cm}$ but not exceeding the equivalent area of the right upper zone. Category $\mathrm{C}$ was defined as being larger than category $\mathrm{B}$ in terms of the sum of the area, with the area exceeding the equivalent area of the right upper zone ${ }^{14)}$.

\section{Outcome measurement}

The outcome of interest was time to progression of chest radiographs, which was calculated by subtracting the year of the patient's first visit at the clinic from the year of the first detection of the progression of the film. Patients were censored at the last year of their visits or on 30 June 2011 if there was no progression of their radiographs. Progression of the disease or radiograph in the present study was defined as an increase in profusion of at least one minor category or new development of a large opacity. In addition, an increase in size of large opacities by at least one category according to the ILO classification system was counted as progression of the disease. For the subgroup assessment, we measured the mean duration of progression from one major category to another and compare them. 


\section{Statistical analysis}

Means [ \pm standard deviation (SDs)] and frequencies (\%) were used to describe patients' characteristics. The Kaplan-Meier test was utilized to estimate the probability of radiographic progression and the median time to progression. The log-rank test was also utilized to compare the median time to progression among different exposed groups. The hazard ratio (HR) was used to describe the ratio of median time to progression between smokers and nonsmokers. In the subgroup analysis, the Kruskal-Wallis method and Wilcoxon rank-sum test were performed to assess mean duration of radiographic progression among major categories. All analyses were performed using Stata version 11. A $p$ value less than 0.05 was considered statistically significant.

\section{Results}

A total of 65 patients who met the inclusion criteria were included in the study. All chest radiographs of each patient were reviewed and compared with the standard ILO films from the first to last year of their visits by the two physicians as mentioned previously. The mean age of the patients at their first visit to the clinic was $58.60 \pm 7.10$ years. The mean duration of dust exposure was $14.72 \pm 7.93$ years. Among the 65 patients, $54(83.08 \%)$ were smokers. The mean duration of follow-up was $16.96 \pm 7.12$ years, with a range from 4-34 years. Other baseline characteristics are shown in Table 1.

The profusion of small opacities in all patients at their first visit was classified by the readers as

Table 1. Baseline characteristics of the study population

\begin{tabular}{ll}
\hline Characteristics & $\begin{array}{l}\text { Patients (N=65) } \\
\text { (Mean } \pm \text { SD) }\end{array}$ \\
\hline Age (years) & \\
First visit & $58.60 \pm 7.10$ \\
Last visit & $75.58 \pm 5.64$ \\
Smoking (n, \%) & \\
Smoker & $54(83.08 \%)$ \\
Nonsmoker & $11(16.92 \%)$ \\
Smoking history (pack-years) & $27.04 \pm 22.66$ \\
Duration of dust exposure (years) & $14.72 \pm 7.93$ \\
Duration of follow-up (years) & $16.96 \pm 7.12$ \\
FEV1 (liters) & \\
First visit & $2.26 \pm 0.52$ \\
Last visit & $1.82 \pm 0.59$ \\
FVC (liters) & \\
First visit & $2.98 \pm 0.58$ \\
Last visit & $2.62 \pm 0.68$ \\
\hline
\end{tabular}

FEV: forced expiratory volume, FVC: forced vital capacity. follows: $1 / 0=13$ cases $(20 \%), 1 / 1=20$ cases $(30.77 \%)$, $1 / 2=5$ cases $(7.69 \%), 2 / 1=12$ cases $(18.46 \%), 2 / 2=13$ cases $(20 \%), 2 / 3=1$ case $(1.54 \%)$ and $3 / 3=1$ case (1.54\%). Large opacities were observed on chest radiographs of 25 patients $(38.46 \%)$ on their first visit. Among these, 3 cases were classified in category A, while the other 22 cases were classified in category B. Category $\mathrm{C}$ was not observed at the beginning of the study.

The incidence rate of radiographic progression was 42 per 1,000 person-years, with a median time to progression of 17.00 years (95\% CI: 12.30-20.46). A survival curve was constructed with the Kaplan-Meier method to describe the time to progression as shown in Fig. 1.

Regarding exposure, the incidence rates in patients who were exposed to silica for up to 15 years and for more than 15 years were 38 and 51 per 1,000 personyears, respectively, with median times to progression of 19.00 and 13.79 years $(p=0.27)$, respectively, as shown in Fig. 2.

Concerning smoking, the incidence rates in smokers and nonsmokers were 47 and 27 per 1,000 personsyears, respectively. The survival curve (Fig. 3) represented the time to progression between smokers and nonsmokers with median times to progression of 14.46 and 19.00 years, respectively $(p=0.21)$. The hazard ratio between smokers and nonsmokers was 1.82 (95\% CI: 0.70-4.74).

Overall, there were 33 cases of radiographic progression. We performed a subgroup analysis in those 33 patients to compare the mean duration of progression from one major category to another. The change in profusion in a major category was analyzed. No matter how many progressions patients had, any change in profusion in a major category was analyzed. For this purpose, category 4 was used to

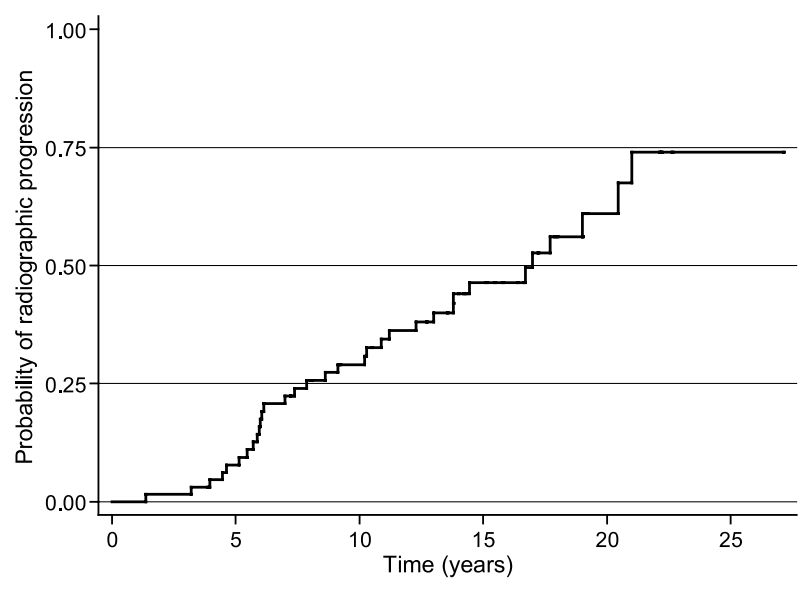

Fig. 1. Probability of radiographic progression of silicosis among workers. 


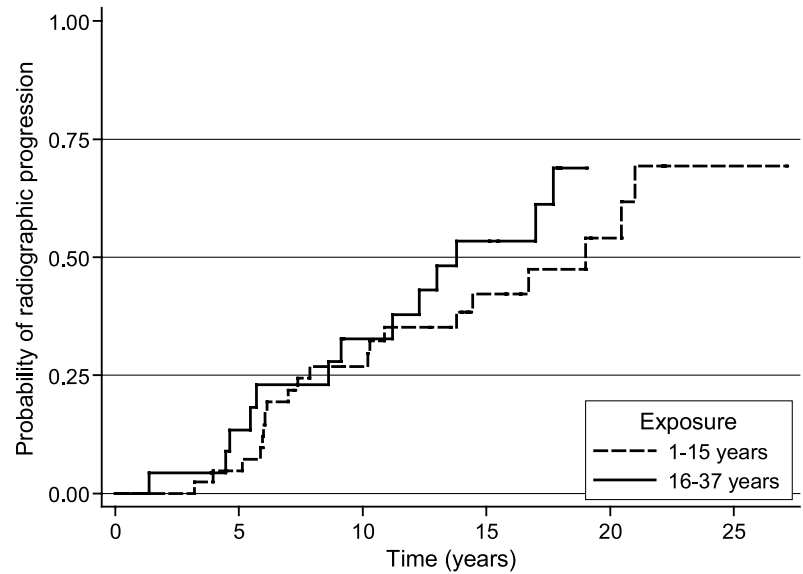

Fig. 2. Probability of radiographic progression of silicosis in workers exposed to silica for up to 15 years and more than 15 years.

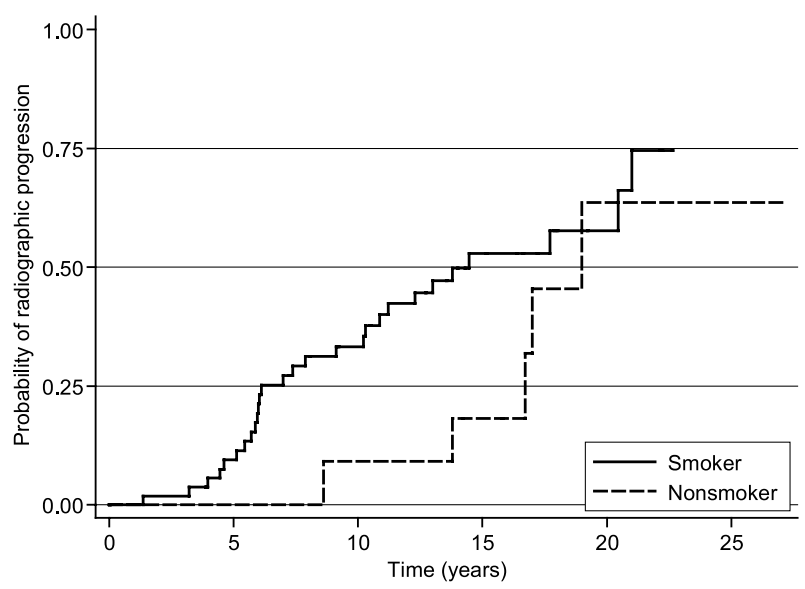

Fig. 3. Probability of radiographic progression of silicosis in smokers and nonsmokers.

represent all categories of large opacities, according to the Japanese Pneumoconiosis Law ${ }^{13)}$. Ultimately, 22 out of 33 cases had radiographic progression in a major category. The mean durations of progression from category 1 to category 2 and from category 2 to category 3 were 5.43 and 3.00 years, respectively, whereas those from category 1 to category 4 and category 2 to category 4 were 14.55 and 10.65 years, respectively (Table 2). Among these cases, 9 cases in category 1 and 10 cases in category 2 had a radiographic change to category 4 directly without changing through category 2 and category 3 , respectively.

\section{Discussion}

The present longitudinal study showed progression of silicosis in terms of CT-proven radiographic findings among Japanese tunnel workers even in the absence of further silica exposure. The incidence rate
Table 2. Numbers and duration of radiographic progression among 22 patients

\begin{tabular}{cccc}
\hline Progression & $\begin{array}{c}\text { Number } \\
(\mathrm{N}=24 *)\end{array}$ & $\begin{array}{c}\text { Mean } \pm \mathrm{SD}^{* *} \\
\text { (years) }\end{array}$ & $\begin{array}{l}\text { Range } \\
\text { (years) }\end{array}$ \\
\hline Category 1 to 2 & 3 & $5.43 \pm 0.29$ & $5.13-5.71$ \\
Category 1 to 4 & 9 & $14.55 \pm 7.54$ & $4.62-31.79$ \\
Category 2 to 3 & 1 & $3.00 \pm 0.00$ & $3.00-3.00$ \\
Category 2 to 4 & 11 & $10.65 \pm 7.20$ & $1.37-21.00$ \\
\hline
\end{tabular}

*Two out of 22 patients had a 2-step progression. ** Statistical analysis was performed by the Kruskal-Wallis method $(p=0.14)$.

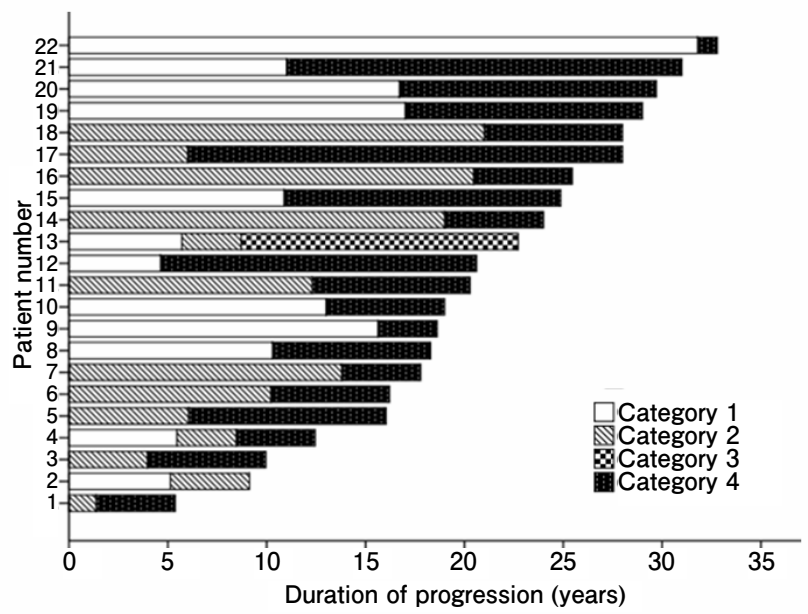

Fig. 4. Duration of radiographic progression in each patient. *Patients No. 4 and No. 13 had a 2-step progression.

of any radiographic progression was 42 per 1,000 person-years, with a median time to progression of 17 years. Even though all subjects of the present study had severed their employment prior to their initial visit to the Kinro Clinic, results showed progression from simple to complicated stages with radiographic large opacities, or progressive massive fibrosis (PMF) in pathologic terminology. Interestingly, large opacities emerged in the category 1 cases without progression of profusion of small rounded opacities.

The larger the area occupied by large opacities, the worse the symptoms become. Unfortunately, there is no curative treatment except for lung transplantation. As a result, prevention is the best way to eliminate silicosis. Both early detection and termination of silica exposure result in delayed progression ${ }^{21}$. The present study showed progression of silicosis among tunnel workers in the absence of further silica exposure.

Among the previous studies investigating the progression of silicosis, the rate of radiographic progression, which varied in different occupations and 
duration of follow-up, ranged from 24 to $65 \%^{5-12)}$. The rate of radiographic progression of silicosis among tunnel workers in the present study was $51 \%$, with a mean follow-up period of $16.96 \pm 7.12$ years. However, there were some differences in terms of the definition of radiographic progression and methods of radiographic assessment among the studies. In the present study, we interpreted chest radiographs using a side-by-side method, which viewed all films of each case together in known temporal order $^{20)}$. This method is sensitive in detecting radiographic progression of silicosis.

Exposure duration was not associated with radiographic progression in this study, which contradicts the results concerning silicosis among sandblasters ${ }^{5)}$ and slate pencil workers ${ }^{6}$. There are some other reports that could not show such association, which include those concerning silicosis among iron-ore miners $^{7)}$ and silica flour packers ${ }^{12}$. One of the reasons we could not find such association in our study was that our study was a clinic-based retrospective study with possible recall bias. In addition, there was no information about the dust concentration, which may have differed from workplace to workplace. The cumulative exposure, which takes into consideration the silica concentration, should be a better indicator, as shown in the study of silicosis among silica flour packers $^{12}$. Other factors that may affect the analysis are use of respiratory protective equipment and specific tasks at the workplace. Due to the absence of the necessary information, we could not analyze the association between cumulative exposure and radiographic progression.

Smoking was previously investigated as a risk factor for radiographic progression of silicosis among sandblasters $^{5)}$, slate pencil workers ${ }^{6}$, and granite quarry workers ${ }^{9}$. The present study as well as the previous studies could not show the effect of a smoking habit on radiographic progression $(p=0.21)$. Furthermore, we could not demonstrate the effect of dose-dependent cigarette smoking (pack-year) and progression. The effect of second-hand smoke may be one possible explanation, as the prevalence of smoking was very high when subjects were in workplaces in the 1950s-70s. In addition, workers in our study were heavy smokers with a mean of 27 pack-years.

With the emergence of large opacities, simple silicosis progresses to complicated silicosis. This radiographic finding is classified as category 4 according to Japanese Pneumoconiosis Law. Subgroup analysis in the progressed group showed direct progression from simple silicosis with category 1 or 2 to complicated silicosis with large opacities. The mean durations from category 1 to category 4 and category 2 to category 4 were 14.55 and 10.65 years, respectively. In contrast to our study, the rate of radiographic progression from category 1 to category 2 among whetstone cutters, who received compensation, during a 15-year follow-up period was $33 \%$, whereas there was no progression from category 1 to category 3 or $4^{11)}$. Furthermore, the rate of progression from category 2 to category 3 was similar to that from category 3 to category $4(36 \%)$. There was high probability of radiographic progression from category 3 to category 4 , which was as high as $63 \%$. Concerning duration of progression, American coal miners who received compensation were reported to have a relatively short mean duration of 12 years between the last normal radiograph and the presentation of $\mathrm{PMF}^{22)}$.

The prevalence of cases in category 4 in the present study, which accounted for $38 \%$ of cases at the first visit and increased to $66 \%$ of cases at the end of the study, was relatively high when compared with that in the previous study conducted among whetstone cutters during a 40 -year follow-up period, which was $6 \%$ and subsequently increased to $14 \%{ }^{11)}$. In addition, there was evidence to support the premise that continuation of silica exposure after diagnosis of silicosis was associated with advanced radiographic progression ${ }^{21}$. It should be stated that it is important to followup tunnel workers with silicosis for a long period in order to delay the onset of or, at least, provide early detection of complicated silicosis, which probably comes with other associated diseases such as tuberculosis, COPD, pulmonary hypertension, lung cancer, etc.

It is difficult to conduct a longitudinal study among tunnel workers because most of them migrate from place to place to work. There is also limited access to information after they quit their jobs or retire. At the clinic, silicotic patients receive medical attention at least once a year. As a result, we could retrospectively review and assess all chest radiographs. Considering the duration of a follow-up over a period of 4 to 34 years with an average of 17 years, this study could observe patients for a relatively longer period than could other studies $5,6,8,9,12)$. To the best of our knowledge, the present study is the first longitudinal study investigating radiographic progression of silicosis among tunnel workers.

Although a relatively greater number of subjects were analyzed in the present study compared with longitudinal studies of other occupational groups ${ }^{5,12)}$, chance may have the effect of obscuring the association of the factors among the groups, i.e., smoking and exposure. Selection bias may have led us to underestimate the progression of silicosis, as we could not include the deceased or patients admitted to hospital due to complication and worsening of respiratory function in our clinic cohort, and these individu- 
als may have had a more severe form of the disease than the subjects in the current study. Consequently, our findings may have postulated the best situation of radiographic progression of silicosis among Japanese tunnel workers. Another limitation of a retrospective study as described previously is that we cannot know the year of onset of silicosis precisely ${ }^{21)}$. It is possible that the onset of the disease may have been several years prior to our diagnosis. In other words, the period between the actual onset of the disease and the first visit at the clinic is not certain. Even with these limitations, the present retrospective observation of silicotic patients revealed an unusual progression pattern of radiographic findings from category 1 , which is the lowest profusion, to category 4, a synonym for complicated silicosis, without passing through category 2 or 3 .

In conclusion, our findings showed that silicosis progressed at a relatively high rate among tunnel workers who were followed up for up to 34 years even after silica exposure had been discontinued. It is necessary to regularly follow-up patients for a long period. In addition, progression from category 1 directly to category 4 may require further investigation not only for better understanding of the disease but also for improvement of disease prevention.

Acknowledgments: The authors would like to thank Drs.Prasert Assantachai and Ammarin Thakkinstian and the staff of the Department of Preventive and Social Medicine, Faculty of Medicine Siriraj Hospital; Section for Clinical Epidemiology and Biostatistics, Faculty of Medicine Ramathibodi Hospital; Department of Environmental Medicine, Kochi Medical School; and Kinro Clinic for their support during this study.

\section{References}

1) World Health Organization. Elimination of silicosis. GOHNET Newsletter. [Online]. 2007 [cited 2012 Oct 11]; Available from: URL: www.who. int/entity/occupational_health/publications/newsletter/ gohnet12e.pdf

2) International Agency for Research on Cancer. IARC Working Group on the Evaluation of Carcinogenic Risks to Humans: Silica, Some Silicates, Coal Dust and Para-Aramid Fibrils. [Online]. 1997 [cited 2012 Oct 11]; Available from: URL: http://monographs. iarc.fr/ENG/Monographs/vol68/mono68.pdf

3) Kimura K, Ohtsuka Y, Kaji H, et al. Progression of pneumoconiosis in coal miners after cessation of dust exposure: a longitudinal study based on periodic chest X-ray examinations in Hokkaido, Japan. Intern Med 2010; 49: 1949-56.

4) Kang SK, Kim EA. Occupational diseases in Korea. J Korean Med Sci 2010; 25: S4-12.
5) Hughes JM, Jones RN, Gilson JC, et al. Determinants of progression in sandblasters' silicosis. Ann Occup Hyg 1982; 26: 701-12.

6) Saiyed HN, Chatterjee BB. Rapid progression of silicosis in slate pencil workers: II. A follow-up study. Am J Ind Med 1985; 8: 135-42.

7) Jorgensen HS. Silicosis in the iron-ore mine in Kiruna, Sweden, and the future need for silicosis control. Int Arch Occup Environ Health 1986; 58: 251-7.

8) $\mathrm{Ng} \mathrm{TP}$, Chan SL, Lam KP. Radiological progression and lung function in silicosis: a ten year follow up study. Br Med J 1987; 295: 164-8.

9) Lee HS, Phoon WH, Ng TP. Radiological progression and its predictive risk factors in silicosis. Occup Environ Med 2001; 58: 467-71.

10) Miller BG, Hagen S, Love RG, et al. Risks of silicosis in coalworkers exposed to unusual concentrations of respirable quartz. Occup Environ Med 1998; 55: 52-8.

11) Ogawa $S$, Imai H, Ikeda M. A 40-year follow-up of whetstone cutters on silicosis. Ind Health 2003; 41: 69-76.

12) Mohebbi I, Zubeyri T. Radiological progression and mortality among silica flour packers: a longitudinal study. Inhal Toxicol 2007; 19: 1011-7.

13) Ministry of Labour, the Government of Japan. Pneumoconiosis Law. [Online]. 1999 [cited 2012 Oct 11]; Available from: URL: http://www.jniosh.go.jp/ icpro/jicosh-old/english/law/Pneumoconiosis_Law/ index.html

14) International Labour Office. Guidelines for the use of the ILO International Classification of Radiographs of Pneumoconioses. [Online]. 2011 [cited 2012 Oct 11]; Available from: URL: http://www.ilo.org/safework/info/publications/ WCMS_168260/lang--en/index.htm

15) National Institute for Occupational Safety and Health. NIOSH B Reader Program. [Online]. 2011 [cited 2012 Oct 11]; Available from: URL: http:// www.cdc.gov/niosh/topics/chestradiography/breaderinfo.html

16) Zhou H, Kusaka Y, Tamura T, et al. The 60-film set with 8-index for examining physicians' proficiency in reading pneumoconiosis chest X-rays. Ind Health 2012; 50: 84-94.

17) Zhou H, Kusaka Y, Tamura T, et al. Proficiency in reading pneumoconiosis radiographs examined by the 60 -film set with 4 -factor structuring 8 -index. Ind Health 2012; 50: 142-6.

18) Suganuma N, Kusaka Y, Hering KG, et al. Selection of reference films based on reliability assessment of a classification of high-resolution computed tomography for pneumoconioses. Int Arch Occup Environ Health 2006; 79: 472-6.

19) Suganuma N, Kusaka Y, Hering KG, et al. Reliability of the proposed international classification of high-resolution computed tomography for occupational and environmental respiratory diseases. J Occup Health 2009; 51: 210-22. 
20) Liddell FDK. Assessment of radiological progression of simple pneumoconiosis in individual miners. Br J Ind Med 1974; 31: 185-95.

21) Carneiro AP, Barreto SM, Siqueira AL, Cavariani F, Forastiere F. Continued exposure to silica after diagnosis of silicosis in Brazilian gold miners. Am J Ind
Med 2006; 49: 811-8.

22) Wade WA, Petsonk EL, Young B, Mogri I. Severe occupational pneumoconiosis among West Virginian coal miners: one hundred thirty-eight cases of progressive massive fibrosis compensated between 2000 and 2009. Chest 2011; 139: 1458-62. 